\title{
The application of a multi-domain boundary face method in a steady state heat conduction problem
}

\author{
Jianming Zhang, Xianghe Li \& Yuan Li \\ State Key Laboratory of Advanced Design and Manufacturing \\ for Vehicle Body, Hunan University, China
}

\begin{abstract}
This paper presents a multi-domain boundary face method (MD-BFM) for solving steady-state heat conduction problems on large-scale engineering structures. In the BFM, both boundary integration and variable approximation are performed in the 2-D parametric space of the boundary surfaces of the body in question. The surface parameters are obtained through the boundary representation data structure (B-rep) implanted in all CAD packages. The geometric quantities in the boundary integrals of the BIE are computed directly in the parametric spaces of the surfaces to avoid geometric errors. On the other hand, a MDBFM is necessary for structures of complex shapes or constituted of different kinds of materials. In this work, the multi-domain BFM is developed and applied for heat conduction analysis of a real gravity dam. Numerical results have demonstrated that our method can achieve comparable accuracy than other methods (e.g. the FEM), but at much lower costs in terms of both computer resources and human labor.
\end{abstract}

Keywords: boundary face method, steady state heat conduction, multi-domain problem, large scale problem.

\section{Introduction}

Problems of heat conduction find important application in engineering. Many numerical methods have been proposed to solve these problems, such as the finite difference method (FDM) [1, 2], the finite volume method (FVM) [3, 4], the finite element method (FEM) $[5,6]$ and the boundary element method (BEM) $[7,8]$. 
In practical analysis, structures of complex shapes are usually of very large size. The computation scale of thermal analysis by FEM implementation will exceed hundreds of millions degrees, and this level of analysis cannot be performed in micro-computers. Because of the computational scale limitation, the overall analysis on the dam body by FEM is usually unavailable. In practical applications, many special elements including multi-layer element are usually employed $[9,10]$. The employment of these special elements, however, will inevitably introduce errors.

In the conventional BEM implementation of structural analyses, a geometric model is firstly built with a CAD package. Then, using a meshing tool, the geometric model is converted into a discrete model. The CAD and BEM are treated as separate modulus requiring different methods and representations [11], which include continuous parametric models and discrete models, respectively. In BEM, the elements are used for boundary integration and approximation of geometry. Once the BEM model is constructed from CAD, the information of geometry is only derived from standard elements. Therefore, geometric errors are introduced. Moreover, the link between BEM model and CAD system is often difficult to obtain, thus making it difficult to carry out adaptive mesh refinement [12].

To cope with the problems above, we have developed a boundary integral equation (BIE) based method, which is called the boundary face method (BFM) [13-15]. In BFM, both boundary integration and variable approximation are performed on boundary faces, which are represented in parametric form exactly as the boundary representation data structure in most CAD systems. The parametric surface, which encapsulates the exact geometry of corresponding face, is discretized by surface elements in parametric space. These elements are used for the boundary integration and variable approximation. For boundary integration, however, the geometric data at Gaussian quadrature points, such as the coordinates, the Jacobian and the outward normal are calculated directly from the faces rather than elements, thus no geometric error will be introduced. The direct boundary integration and approximation in parametric space of surfaces forms an intrinsic feature of the BFM when compared with the conventional BEM.

Many implementations of the BFM can be found. Qin et al. [16] implement the BFM by employing a finite element scheme. In Qin's implementation, the element was defined in the parametric space of the boundary surfaces. The mesh was also generated in the parametric space. Gu et al. [17] used a B-spline interpolation scheme for physical variables approximation in the BFM. And by employing the same interpolation function as in the geometric modeling, $\mathrm{Gu}$ et al. [18] performed a iso-geometric analysis based on the BFM. Zhuang et al. [19] implemented the BFM on the geometric models which are constructed through the subdivision surfaces. By introducing a specified element, the BFM is applied for analysis of structures containing tubular cavities [20].

The BFM was also extended for many engineering problems. By combining with the dual reciprocity, Zhou et al. [21] extended the BFM for the nonhomogeneous heat conduction problem and non-homogeneous elasticity 
problem. To solve the transient heat conduction problem, Zhou et al. coupled the BFM and the convolution quadrature method. The transient heat conduction problem was also solved by Guo et al., using a Laplace transformation method and the multiple reciprocity BFM. The BFM was extended for acoustic problems by Wang et al. For large scaled problem, Wang et al. coupled the BFM and the fast multiple method (FMM) to accelerate the computation.

All the mentioned application was based on problem containing a single domain. Engineering structures, however, are usually very complex and should be divided into many subdomains in actual analysis. This paper extends the BFM for steady-state heat conduction problems on multi-domain models. The matrix assembling is of great importance in the multi-domain computation. The method for assembling three domains which are connected by each other will be described in this paper. As an illustration example, a steady state heat conduction analysis on a real gravity dam will be performed by the multi-domain BFM. The considering structure will also be analyzed by the finite element method which is conducted in the ABAQUS. A comparative study between results obtained by both methods will be made.

This paper is organized as follows: Sec. 2 describes the BFM for steady-state heat conduction problem. The following section illustrates the multi-domain BFM and the matrices assembling in detail. The numerical example is presented in Sec. 4. This paper ends with conclusions and some future work.

\section{Boundary face method for 3D steady heat conduction}

\subsection{Boundary integral equation}

The steady heat conduction problem in three dimensions can be mathematically described as

$$
\begin{aligned}
u,_{i i} & =0, \quad \forall x \in \Omega \\
u & =\bar{u}, \quad \forall x \in \Gamma_{u} \\
u_{,_{i}} n_{i} & \equiv q=\bar{q}, \quad \forall x \in \Gamma_{q}
\end{aligned}
$$

where $\Omega$ is the corresponding domain which is enclosed by $\Gamma=\Gamma_{u} \cup \Gamma_{q}$. On the essential boundary $\Gamma_{u}$ and the flux boundary $\Gamma_{q}$, we impose the boundary condition $\bar{u}$ and $\bar{q}$, which denote the prescribed temperature and the normal flux, respectively. $n$ with components $n_{i}, i=1,2,3$ is the outward normal on the boundary $\Gamma$.

The problem can be converted into an equivalent BIE which is described as the following formulation

$$
\int_{\Gamma}(u(\mathbf{s})-u(\mathbf{y})) q^{s}(\mathbf{s}, \mathbf{y}) d \Gamma=\int_{\Gamma} q(\mathbf{s}) u^{s}(\mathbf{s}, \mathbf{y}) d \Gamma
$$

In this formula, y and $\mathrm{s}$ stand for the field point and source point, respectively. $q=\partial u / \partial n$ is the boundary normal flux. $\mathrm{u}^{\mathrm{s}}(\mathrm{s}, \mathrm{y})$ and $\mathrm{q}^{\mathrm{s}}(\mathrm{s}, \mathrm{y})$ are the corresponding fundamental solutions which satisfy

$$
u^{s}{ }_{i i}=\delta(y, s), \quad \forall s \in \Omega
$$


and

For 3-D problems,

$$
q^{s}=\partial u^{s} / \partial n(s)
$$

$$
u^{s}(\mathbf{s}, \mathbf{y})=\frac{1}{4 \pi} \frac{1}{r(\mathbf{s}, \mathbf{y})}
$$

where $r$ is the distance between $y$ and $s$.

\subsection{Boundary face method}

In this sub-section, an effective Lagrange approximation method, which is based on the surface element, is presented. The surface element is defined in 2-D parametric space of the surface rather than in the physic space or other parametric spaces. Thus the geometric components in the considered boundary integral can be computed directly through a parametric transformation. And this parametric transformation is the same as the mapping scheme in the parametric surface, in other word, the geometric quantities in the integral are exactly obtained. A four-node quadrilateral surface element is taken as an illustration example of the physical variable approximation.
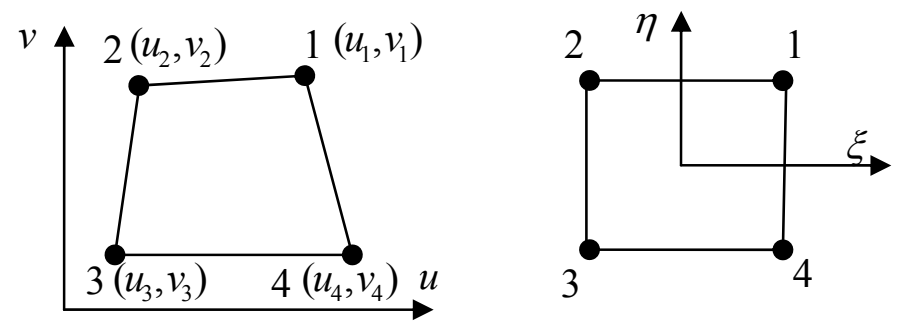

Figure 1: $\quad$ Four-node surface element and coordinate mapping.

The corresponding shape functions we construct in this implementation are

$$
\begin{aligned}
& N_{1}=\frac{1}{4}(1+\xi)(1+\eta), N_{2}=\frac{1}{4}(1-\xi)(1+\eta) \\
& N_{3}=\frac{1}{4}(1-\xi)(1-\eta), N_{4}=\frac{1}{4}(1+\xi)(1-\eta)
\end{aligned}
$$

It should be emphasized here that, these shape functions are only applied to approximate the physical variables on the boundary surfaces. The geometric quantities keep exact in this implementation, and this is the main difference from the standard BEM.

$$
\begin{aligned}
& u(x, y, z)=u(u, v)=u(\xi, \eta)=\sum_{k=1}^{N} N_{k}(\xi, \eta) u_{k} \\
& q(x, y, z)=u(u, v)=u(\xi, \eta)=\sum_{k=1}^{N} N_{k}(\xi, \eta) q_{k}
\end{aligned}
$$

where $\mathrm{x}=\mathrm{x}(\mathrm{u}, \mathrm{v}), \mathrm{y}=\mathrm{y}(\mathrm{u}, \mathrm{v})$ and $\mathrm{z}=\mathrm{z}(\mathrm{u}, \mathrm{v}), \mathrm{u}_{\mathrm{k}}$ and $\mathrm{q}_{\mathrm{k}}$ are values of temperature and the normal flux on boundary nodes, $\mathrm{N}$ is the total number of interpolating points. 
With this approximation scheme, Eq. (2) is discretized into:

$$
\sum_{j=1}^{M} \int_{\Gamma_{j}} u^{s}(\mathbf{s}, \mathbf{y}) \sum_{k=1}^{N} N_{k}(\mathbf{s}) q_{k} d \Gamma(\mathbf{s})-\sum_{j=1}^{M} \int_{\Gamma_{j}} q^{s}(\mathbf{s}, \mathbf{y}) \sum_{k=1}^{N}\left(N_{k}(\mathbf{s})-N_{k}(\mathbf{y})\right) u_{k} d \Gamma(\mathbf{s})=0
$$

After we collocate the field point at every interpolation point, we will get the following system:

$$
\mathbf{G q}-\mathbf{H u}=\mathbf{0}
$$

in which

$$
H_{i k}=\sum_{j=1}^{M} \int_{\Gamma_{j}} q^{s}\left(\mathbf{s}, \mathbf{y}_{i}\right)\left(N_{k}(\mathbf{s})-N_{k}\left(\mathbf{y}_{i}\right)\right) d \Gamma(\mathbf{s})
$$

and

$$
G_{i k}=\sum_{j=1}^{M} \int_{\Gamma_{j}} u^{s}\left(\mathbf{s}, \mathbf{y}_{i}\right) N_{k}(\mathbf{s}) d \Gamma(\mathbf{s})
$$

\section{The multi-domain boundary face method}

In this section, we concern the multi-domain boundary face method for steady state heat conduction problem. Three cubes are illustrated as examples in Fig. 2.

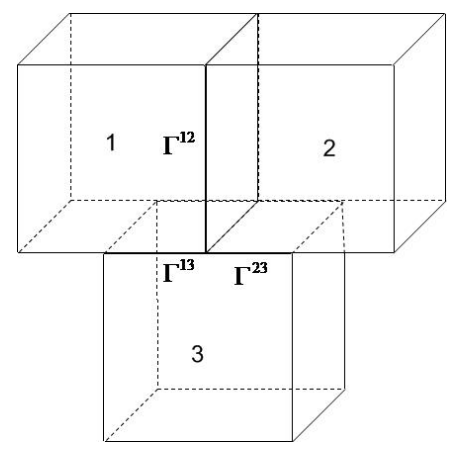

Figure 2: $\quad$ The sketch map of 3 cubes.

In this model, cube 1 and cube 2 intersect in $\Gamma^{12}$, cube 1 and cube 3 intersect in $\Gamma^{13}$ and cube 2 and cube 3 intersect in $\Gamma^{23}$. On the intersection boundaries, we have the condition:

$$
\widehat{u}_{i}=\widehat{u}_{j}
$$

and

$$
\widehat{q}_{i}=-\widehat{q}_{j}
$$

For cube 1 , the boundary integral equations are: 


$$
\left[\begin{array}{lllll}
H_{d d} & H_{d n} & H_{d r} & H_{d 2} & H_{d 3} \\
H_{n d} & H_{n n} & H_{n r} & H_{n 2} & H_{n 3} \\
H_{r d} & H_{r n} & H_{r r} & H_{r 2} & H_{r 3} \\
H_{2 d} & H_{2 n} & H_{2 r} & H_{22} & H_{23} \\
H_{3 d} & H_{3 n} & H_{3 r} & H_{32} & H_{33}
\end{array}\right]\left\{\begin{array}{c}
\bar{u} \\
u \\
\tilde{u} \\
\hat{u}_{2} \\
\hat{u}_{3}
\end{array}\right\}=\left[\begin{array}{lllll}
G_{d d} & G_{d n} & G_{d r} & G_{d 2} & G_{d 3} \\
G_{n d} & G_{n n} & G_{n r} & G_{n 2} & G_{n 3} \\
G_{r d} & G_{r n} & G_{r r} & G_{r 2} & G_{r 3} \\
G_{2 d} & G_{2 n} & G_{2 r} & G_{22} & G_{23} \\
G_{3 d} & G_{3 n} & G_{3 r} & G_{32} & G_{33}
\end{array}\right]\left\{\begin{array}{c}
q \\
\bar{q} \\
\tilde{q} \\
\hat{q}_{2} \\
\hat{q}_{3}
\end{array}\right\}
$$

in which the symbols $d, n, r$ stand for the boundary conditions of Dirichlet type, Neumann type and Robin type, respectively. Subscripts 2 and 3 denote for the areas where the boundary of cube 1 intersects with cube 2 or cube 3 . By considering the boundary condition, we reassemble the above boundary integral systems into:

$$
\left[\begin{array}{ccccccc}
G_{d d}^{1} & -H_{d n}^{1} & \left(-H_{d r}^{1}-G_{d r}^{1} \alpha\right) & -H_{d 2}^{1} & -H_{d 3}^{1} & G_{d 2}^{1} & G_{d 3}^{1} \\
G_{n d}^{1} & -H_{n n}^{1} & \left(-H_{n r}^{1}-G_{n r}^{1} \alpha\right) & -H_{n 2}^{1} & -H_{n 3}^{1} & G_{n 2}^{1} & G_{n 3}^{1} \\
G_{r d}^{1} & -H_{r n}^{1} & \left(-H_{r r}^{1}-G_{r r}^{1} \alpha\right) & -H_{r 2}^{1} & -H_{r 3}^{1} & G_{r 2}^{1} & G_{r 3}^{1} \\
G_{2 d}^{1} & -H_{2 n}^{1} & \left(-H_{2 r}^{1}-G_{2 r}^{1} \alpha\right) & -H_{22}^{1} & -H_{23}^{1} & G_{22}^{1} & G_{23}^{1} \\
G_{3 d}^{1} & -H_{3 n}^{1} & \left(-H_{3 r}^{1}-G_{3 r}^{1} \alpha\right) & -H_{32}^{1} & -H_{33}^{1} & G_{32}^{1} & G_{33}^{1}
\end{array}\right]\left\{\begin{array}{l}
q_{d}^{1} \\
u_{n}^{1} \\
\tilde{u}_{r}^{1} \\
\hat{u}_{2}^{1} \\
\hat{u}_{3}^{1} \\
\hat{q}_{2}^{1} \\
\hat{q}_{3}^{1}
\end{array}\right\}=\left[\begin{array}{lll}
H_{d d}^{1} & -G_{d n}^{1} & -G_{d r}^{1} \\
H_{n d}^{1} & -G_{n n}^{1} & -G_{n r}^{1} \\
H_{r d}^{1} & -G_{r n}^{1} & -G_{r r}^{1} \\
H_{2 d}^{1} & -G_{2 n}^{1} & -G_{2 r}^{1} \\
H_{3 d}^{1} & -G_{3 n}^{1} & -G_{3 r}^{1}
\end{array}\right]\left\{\begin{array}{c}
\bar{u}_{d}^{1} \\
\bar{q}_{n}^{1} \\
\beta^{1}
\end{array}\right\}=\bar{y}^{1}
$$

The same as cube 1, for cube 2 and cube 3, we also have the following reassembled boundary integral systems:

Considering the interacting condition and denoting $F_{i r}^{k}=H_{i r}^{k}+G_{i r}^{k} \alpha$, we have the final systems for solution:

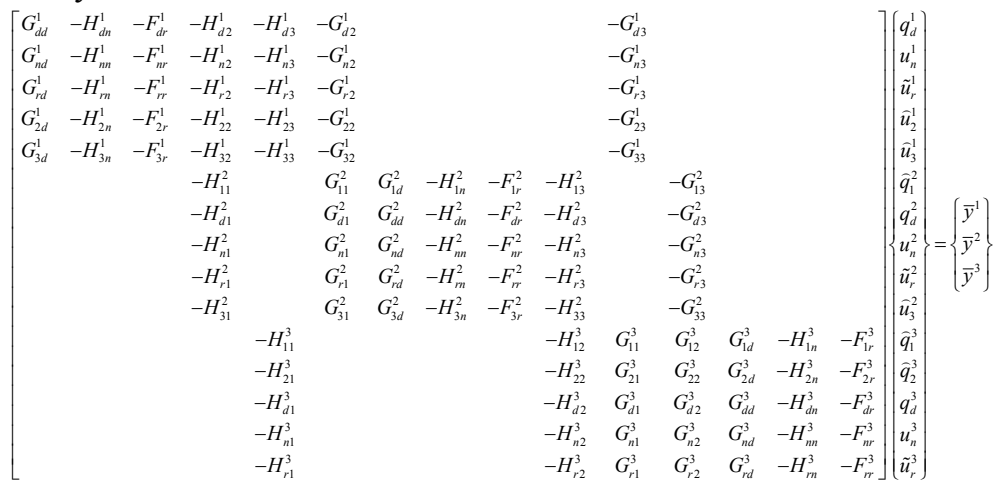

\section{Numerical examples}

In this section, we solved a steady-state conduction problem of a massive gravity dam with the BFM and FEM (obtained from ABAQUS), and made comparisons between them. 


\subsection{Geometry of the model}

Fig. 3 shows the cross-section of the dam. To reduce the computational scale, only a part of the dam (a thin piece with width of 20 meters) is modeled. The computational model consists of two parts: the dam body and the rock foundation.

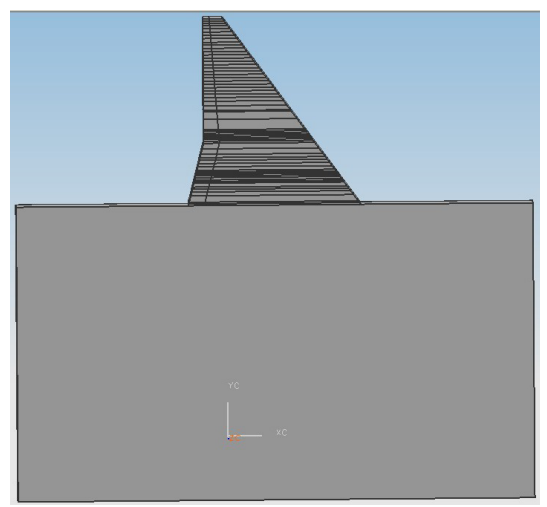

Figure 3: $\quad$ Model of a gravity dam.

The height of the dam body is 171 meters, and the width of the part touching with rock foundation on the first concrete layer is 157 meters. The gradient for downstream slope is 1:0.73. The length and the height of the rock foundation are $471 \mathrm{~m}$ and $157 \mathrm{~m}$ respectively. Besides, the dam body was divided into 62 layers, each layer has various thicknesses.

\subsection{Material properties}

Table 1 provides summaries for the properties of roller concrete and normal concrete, respectively. The material of the first two layers is roller concrete. The other layers are normal concrete. Moreover, it is worth noticing that the material of the rock foundation is assumed to be roller concrete.

Table 1: $\quad$ Properties of roller concrete and normal concrete.

\begin{tabular}{|c|c|c|c|}
\hline & $\begin{array}{c}\text { Heat conductivity } \\
\mathrm{kJ} /(\mathrm{m} \cdot \mathrm{h} \cdot)\end{array}$ & $\begin{array}{c}\text { Specific heat } \\
\mathrm{kJ} /\left(\mathrm{kg} \cdot \mathrm{C}^{\mathrm{o}}\right)\end{array}$ & $\begin{array}{c}\text { Density } \\
\mathrm{kg} / \mathrm{m}^{3}\end{array}$ \\
\hline Roller concrete & 9.27 & 0.9672 & 2400 \\
\hline Normal concrete & 8.766 & 0.9672 & 2450 \\
\hline
\end{tabular}

\subsection{Boundary condition}

There are three kinds of boundary conditions in this analysis. First, boundary condition of fixed air temperature which is the annual average temperature. Second, boundary condition of water temperature. Third, adiabatic boundary that 
there is no change in temperature in the direction normal to the planes, i.e.: $\partial T / \partial n=0.0$.

Adiabatic boundary condition is imposed on the faces of the right and left sides, the bottom of the rock foundation, the front and back sides along the river direction. The upstream water level is $162 \mathrm{~m}$. Areas above the height of 375 meters, the top faces and downstream areas on dam body, right side on rock foundation are exposed to atmosphere. Boundary condition of water temperature is shown below:

1. A deep water temperature of $13.4^{\circ} \mathrm{C}$ is added to areas that located $123.4 \mathrm{~m}$ below water face.

2. A linear function is used in shallow water area. The fitting function is:

$$
T_{w}=20.7-0.0591572 * h
$$

\subsection{The mesh model}

In FEM analysis, we define hexahedral elements consisting of 16 nodes per element. The total number of elements is 47432 , with 272300 computing nodes (see Fig. 4(a)). In BFM, there are 6494 quadratic elements consisting of 8 nodes per element (including triangular and quadrilateral elements) and 29139 computing nodes (see Fig. 4(b))

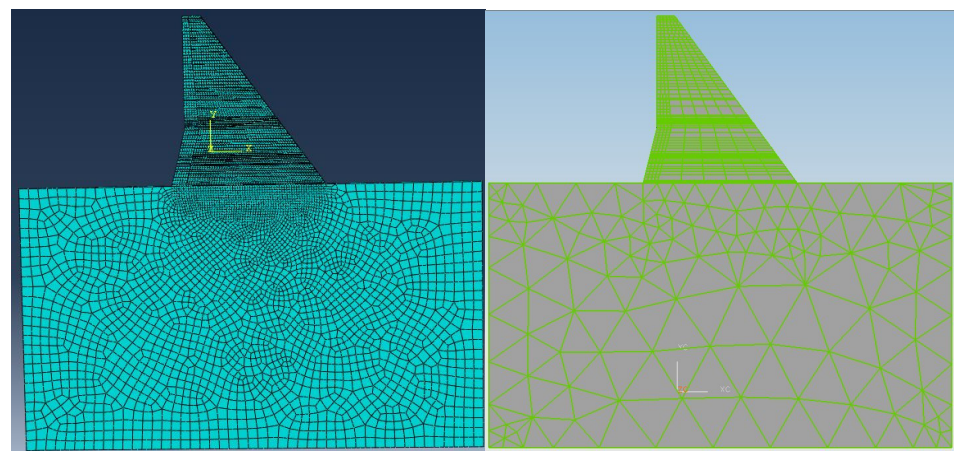

(a) Mesh model of FEM

(b) Mesh model of BFM.

Figure 4: $\quad$ The comparison of mesh model of FEM and BFM.

\subsection{Comparison between FEM and BFM}

Fig. 5 shows a comparison of temperature distribution between FEM and BFM.

Comparing the figures, we can see that the computing results of BFM are consistent with the result obtained by ABAQUS. Therefore, we can draw a conclusion that BFM can be applied to analysis of steady-state heat conduction in large-scale engineering problems with good accuracy and high efficiency. Moreover, in our method, human labor is much less than other methods (e.g. the FEM). 


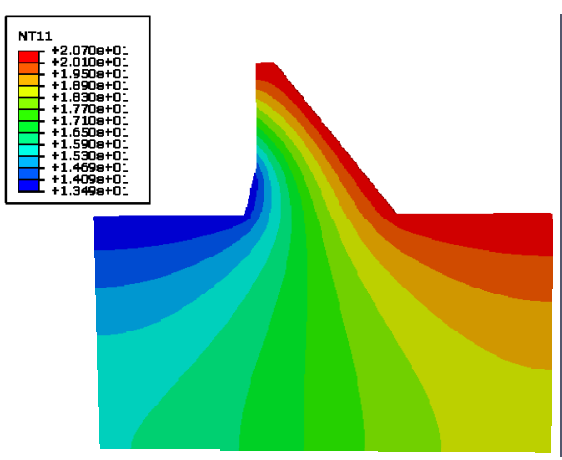

(a) Computing results of FEM

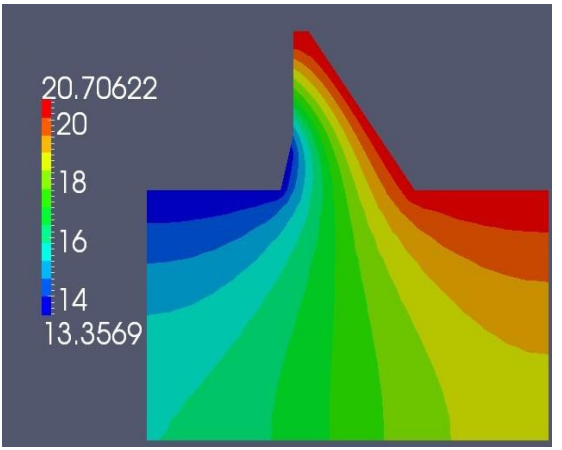

(b) Computing results of BFM

Figure 5: Comparison between FEM and BFM.

\section{Conclusions and future work}

The steady heat conduction problem on a dam body which consists of 62 layers is solved by the multi-domain BFM. The solution is demonstrated and compared with the FEM implementation. The comparison illustrates that the BFM is very accurate in computations of temperature and is suitable for steady-state thermal analysis in dam construction.

For further study, a transient heat conduction problem on multi-domains will be considered. Further accelerating the computation by optimization of domain matrices assembling will also be studied in our future work.

\section{References}

[1] Jaluria Y., Torrance K.E., Computational heat transfer. Hemisphere Publication Corporation, Washington, 1986.

[2] Anderson D.A., Tannehill J.C., Pletcher R.H., Computational fluid mechanics and heat transfer, Hemisphere Publication Corporation, Washington, 1997.

[3] Versteeg H.K., Malalasekera W., An introduction to computational fluid dynamics, The finite volume method, Longman Scientific and Technical: Essex, 1995.

[4] Tao W.Q., Recent advances in computational heat transfer. Science Press: Beijing, 2000.

[5] Reddy J.N., Gartling D.K., The finite element method in heat transfer and fluid dynamics, CRC Press, 2010.

[6] Lewis R.W., Morgan K., Thomas H.R., et al., The finite element method in heat transfer analysis: Wiley, 1996.

[7] Zhou F.L., Xie G.Z., Zhang J.M., et al., Transient heat conduction analysis of solid with small open-ended tubular cavities by boundary face method. Engineering Analysis with Boundary Elements, 37, 2013. 
[8] Wang C.H., Grigoriev M.M., Dargush G.F., A fast multi-level convolution boundary element method for transient diffusion problems. International Journal for Numerical Methods in Engineering, 62, 2005.

[9] Zhu, B.F. and Xu, P., Zone-merged algorithm and zoned different-steplength algorithm in the concrete simulation calculation. Water Power, 6, 1996.

[10] Chen, Y.C., Wang, C.J., Li, S.Y., Wang, R.J. and He, J. 2001, Simulation analysis of thermal stress of RCC dams using 3-D nite element relocating mesh method. Advances in Engineering Software, 21(16).

[11] Kagan P, Fischer A. Integrated mechanically based CAE system using BSpline finite elements. Comput-Aid Design, 32, 2000.

[12] Hughes T.J.R., Cottrell J.A., Bazilevs Y. Isogeometric analysis: CAD, finite elements, NURBS, exact geometry and mesh refinement. Comput Methods Appl Mech Eng, 194, 2005.

[13] Zhou, F.L., Zhang, J.M., Sheng, X.M. and Li, G.Y., Shape variable radial basis function and its application in dual reciprocity boundary face method. Engineering Analysis with Boundary Element, 35(9), 2011.

[14] Qin, X.Y., Zhang, J.M., Li, G.Y. and Sheng, X.M., A finite element implementation of the boundary face method for potential problems in three dimensions. Engineering Analysis with Boundary Element, 34(10), 2010,

[15] Zhang, J., Qin, X., Han, X. and Li, G., A boundary face method for potential problems in three dimensions. International Journal for Numerical Methods in Engineering, 80, pp. 320-337, 2009.

[16] Qin X.Y., Zhang J.M., Li G.Y., Sheng X.M., An element implementation of the boundary face method for 3D potential problems. Engineering Analysis with Boundary Elements, 34, pp. 934-943, 2010.

[17] Gu J.L., Zhang J.M., Sheng X.M., The boundary face method with variable approximation by b-spline basis function. International Journal of Computational Methods, 9, 2012.

[18] Gu J.L., Zhang J.M., Li G.Y., Isogeometric analysis in BIE for 3-D potential problem. Engineering Analysis with Boundary Elements, 36, pp: 858-865, 2012.

[19] Zhuang C., Zhang J.M., Qin X.Y., Zhou F.L., Li G.Y., Integration of subdivision method into boundary element analysis. International Journal of Computational Methods, 9, 2012.

[20] Huang C., Zhang J.M., Qin X.Y., Lu C.J., Sheng X.M., Li G.Y., Stress analysis of solids with open-ended tubular holes by BFM. Engineering Analysis with Boundary Elements, 36, 2012.

[21] Zhou F.L., Zhang J.M., Sheng X.M., Li G.Y., A dual reciprocity boundary face method for 3D non-homogeneous elasticity problems. Engineering Analysis with Boundary Elements, 36, 2012. 